\title{
Effect of Royal Jelly Capsule on Quality of Life of Patients with Multiple Sclerosis: A Double-blind Randomized Controlled Clinical Trial
}

\author{
Khodayar Oshvandi ${ }^{1}$, Maryam Aghamohammadi ${ }^{2}$, Farideh Kazemi $^{3}$, Seyedeh Zahra Masoumi ${ }^{3}$, Mehrdokht \\ Mazdeh $^{4}$ and Mehdi Molavi Vardanjani ${ }^{5, *}$ \\ ${ }^{1}$ Full Professor, PhD in Nursing, Mother and Child Care Research Center, Hamadan University of Medical Sciences, Hamadan, Iran \\ 2 MSc in Medical surgical Nursing, Student Research Committee, School of Nursing and Midwifery, Hamadan University of Medical Sciences, Hamadan, Iran \\ ${ }^{3}$ Department of Midwifery, Mother and Child Care Research Center, School of Nursing and Midwifery, Hamadan University of Medical Sciences, Hamadan, Iran \\ ${ }^{4}$ Department of Neurology, Hamadan University of Medical Sciences, Hamadan, Iran \\ ${ }^{5}$ Research Center for Chronic Diseases (Home Care), School of Nursing and Midwifery, Hamadan University of Medical Sciences, Hamadan, Iran
}

* Corresponding author: Mehdi Molavi Vardanjani, Research Center for Chronic Diseases (Home Care), School of Nursing and Midwifery, Hamadan University of Medical Sciences, Hamadan, Iran. Tel: 00989038113876; Email: m.molavi@umsha.ac.ir

Received 2020 August 21; Revised 2020 September 18; Accepted 2020 0ctober 11.

\begin{abstract}
Background: Among the most important chronic diseases is multiple sclerosis (MS) leading to physical disability and decrease in the patients' quality of life.

Objectives: The present study aimed to investigate the effect of the royal jelly capsule on the life quality of patients with MS.

Methods: This randomized clinical trial study was conducted on a total of 100 patients referring to the MS Society of Hamadan, Iran, in 2018. Allocation sequences were determined using block randomization with a block size of 4 , and the subjects were assigned to the intervention and control groups according to a predetermined sequence. The intervention group received one royal jelly capsule (500 mg) daily, and the control group received a placebo capsule for 90 days. The life quality of the patients and their daily activities were evaluated using the specific questionnaire for the life quality of the patients with MS and Barthel Index of Daily Living Activities before and after the intervention. Data analysis was performed by Stata software (version 13) and appropriate statistical tests with a significance level of 0.05 . Results: The comparison of the mean score of the patients' life quality showed that it was higher in the experimental group in the postintervention $(66.51 \pm 15.08)$ than that reported for the control group $(51.31 \pm 15.08)$ after controlling possible confounding factors, which was statistically significant $(\mathrm{P}<0.001)$. Furthermore, Cohen's d $(1.01 ; 95 \% \mathrm{CI}: 0.59-1.42)$ also indicated the intended large effect size of the intervention. The comparison of daily activity status scores between the two groups also demonstrated an improvement in the experimental group (18.76 \pm 1.44$)$, compared to that of the control group (16.96 \pm 3.01$)$, which was statistically significant ( $\mathrm{P}=0.001$ ).

Conclusion: Based on the findings of the current study, it can be concluded that the use of the royal jelly supplement can be effective in the improvement of the life quality of the patients with MS.
\end{abstract}

Keywords: Multiple sclerosis, Quality of life, Royal jelly

\section{Background}

Among the most common neurological diseases is multiple sclerosis (MS) mainly occurring in young adults and more frequently in women (1). As a chronic inflammatory disease, it is identified by demyelinating lesions in the brain, spinal cord, and ophthalmic nerve. It is estimated that 250,000 to 350,000 individuals in the United States of America are diagnosed with MS, and about 200 new cases of MS are diagnosed almost every week. According to the statistics of the Multiple Sclerosis Society of Iran, 30,000 patients with MS are diagnosed in the country about 5,500,000 of whom are members of the Multiple Sclerosis Society $(1,2)$. Hamadan province with a prevalence of 62.5 per 100,000 individuals is one of the high-risk regions for MS in Iran (3).

The patients with this debilitating and chronic disease suffer from many problems. These problems lead to a limitation in patients' participation in health promotion activities, increase in secondary complications, restriction in independent living, and ultimately a negative impact on their quality of life.
Chronic diseases are reported with many changes in physical, psychological, social, and economic dimensions of life quality. The severity, disease duration, and administered drugs have an effect on the quality of life among patients with chronic diseases. Today, the enhancement of life quality is regarded as a significant and fundamental component of social and economic development by many governments (4). According to the literature, these patients have been reported with a lower quality of life than healthy individuals. Several studies comparing the life quality of these patients and those with other chronic diseases, such as epilepsy, diabetes, rheumatoid arthritis, and inflammatory bowel disease, demonstrated that patients with MS had significantly lower quality of life $(5,6)$.

Although the usual treatment of these patients can decrease the recurrence, severity, and progress of the disease, it has a minimal effect on signs, functional recovery, and life quality of these patients; therefore, they usually seek different methods of treatment (7). Since the last decade, there has been an interest in complementary and alternative medicine in health 
care professionals around the world, and a part of nursing care is increasingly dedicated to complementary and alternative medicine (8). Currently, many patients use a variety of complementary therapies, including herbal medicine, acupuncture, hypnosis, energy therapy, and yoga, to treat and control the symptoms of this disease (9). One of the methods of complementary medicine has been the use of bees and their products. A history of using bees and their productions in the treatment of various diseases goes back to human awareness of the benefits of this insect (8).

One of the bee products is royal jelly which is full of minerals and vitamins B, E, and sex hormones, and its gamma-globin strengthens the immune system. In addition, it is useful for the treatment of weakness, weight loss, and reduction of anorexia, stress, and insomnia. Since apitherapy has been used in many cultures, bee products, such as royal jelly, are used for the treatment of a range of diseases (e.g., arthritis, MS, central and peripheral nervous system diseases, in addition to heart and blood system diseases). Bee toxin is a complicated combination of a range of peptides and proteins with strong neurological and immune effects. Bee toxin consists of 18 active agents, such as melittin (40-50\%) as the main cause of anti-inflammatory, antibacterial, and antiviral activities and apamine (2-3\%) raising cortisol production in the adrenal glands. The results of studies have shown that royal jelly improves diabetic and burn wounds and enhances memory function and resistance to fatigue $(10,11)$.

There have been different studies carried out on the assessment of the impact of royal jelly on different systems of the laboratory animal body. However, a limited number of studies have been conducted on the effects of this substance on the human body. A study performed by Morita et al. showed that a 6-month consumption of royal jelly in volunteers improved many health indicators, such as the process of red blood cell production, glucose tolerance, and mental status (11). Another study carried out by Meng et al. also showed that the consumption of royal jelly in the elderly prevents physical strength loss (13).

\section{Objectives}

Considering the limited number of studies conducted on the effect of royal jelly on the level of health and life quality of patients with MS in Iran, importance of this issue, and high prevalence of MS in Hamadan, this study aimed to investigate the effect of royal jelly on the life quality of patients with MS referring to the MS Society of Hamadan.

\section{Methods}

This randomized controlled trial study was conducted on a total of 100 patients with MS within September and February 2018. The present study has an ethics code (IR.UMSHA.REC.1397.96) and a research code (9703291646). Based on the obtained data from a study performed by Morita et al. (12) and considering $\alpha$ of 0.05 , power of 0.80, M1 of 75, M2 of 73 , Sd1 and Sd2 of 3, and loss of $5 \%$ using sampsi command in Stata software (version 13), the sample size for each group was calculated at 50 individuals. Before the study, the allocation sequence was determined using block randomization with a block size of 4 . The intervention type was written based on a predetermined sequence inside envelopes in a matte package, and the envelopes were numbered in order. The patients meeting the inclusion criteria received a package and were assigned to one of the control or intervention groups based on the contents inside the envelope in case of completing the written consent form (Figure 1).

The inclusion criteria included the lack of sensitivity to bee products, willingness to participate in the study, score of less than 8 on the Expanded Disability Status Scale, age range of 18-60 years, no pregnancy and breastfeeding in women, and no treatment with other methods of complementary medicine. The exclusion criteria included the incidence of allergy to the medication and lack of willingness to continue participation in the study. The intervention group received a 500-mg capsule of royal jelly daily, and the control group received a placebo capsule for 90 days daily under the supervision of a neurologist. In addition, the patients were visited by a neurologist once a month to be investigated regarding the acceptance of treatment and possible complications. Both groups received their routine treatment according to the neurologist's diagnosis.

The applied instruments in the current study were a demographic information questionnaire (e.g., age, gender, marital status, employment status, education level, disease duration, and place of residence), Multiple Sclerosis Impact Scale (MSIS-29), and Barthel Index of Daily Living Activities. The quality of life questionnaire for MS patients (i.e., MSIS-29) consists of 29 items, with the first 20 items and last 9 items measuring the physical and psychological effects of MS on the patient, respectively. Each item has five options with a score of 1 to 5 . The minimum and maximum possible scores will be 29 and 145, respectively. The scores within 29-58, 58-87, and above 87 indicate the low, moderate, and high quality of life, respectively (14).

The Barthel Index of Daily Living Activities is the most widely used tool by researchers and health care professionals to assess the level of this ability in daily activities. The tool was designed in 1965 in the United States by Barthel and Mahoney Florence for the evaluation of the activities, such as personal cleaning, bathing, eating, using the toilet, going up- 


\section{CONSORT Flow Diagram}

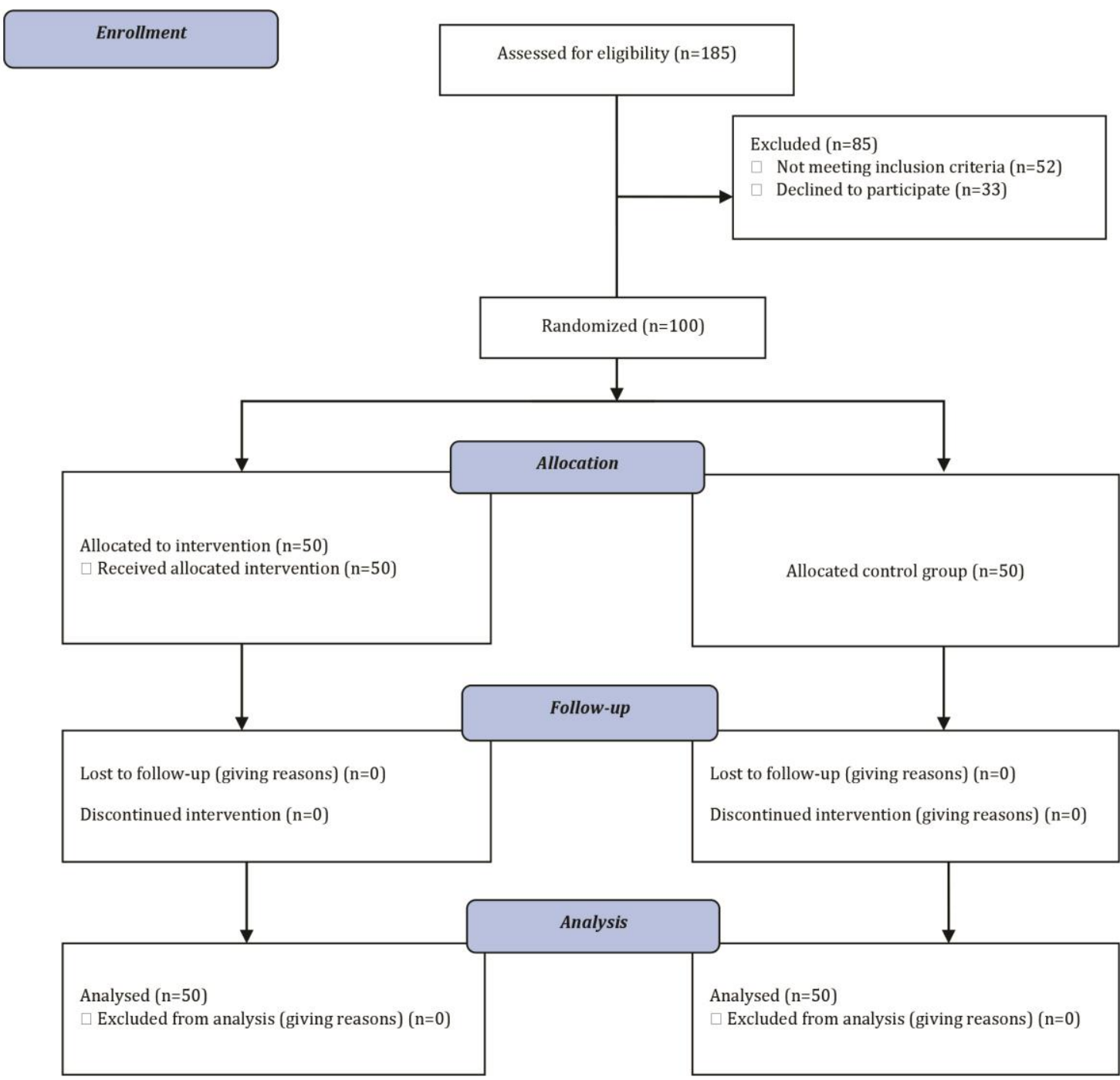

Figure 1. CONSORT flow diagram of selection of study participants

and downstairs, dressing, stool controlling, urine controlling, and walking and mobility. The scoring of the scale includes the inability to obtain a score of 0 , need for significant assistance in obtaining the activity score of 2 , need for moderate assistance in obtaining the activity score of 5 , and need for minimal assistance in obtaining the activity score of 8 and complete independence score of 10 . This tool has 10 items for the scoring of which methods $0-1-2$ have been suggested determining the status of independence or dependence on the performance of daily activities on a scale of 0-20; accordingly, a higher score indicates low dependency in daily activities. The scores of 20,13-19, 12-9, and 8-0 are interpreted as full independence, partial dependence, semi-independent, and complete dependence, respectively (15).

To determine the qualitative content validity of the demographic information questionnaire, 10 faculty members of neurology and nursing were asked to express their opinions on the items, whose opinions were applied at the discretion of the research team. For the evaluation of the reliability of the MSIS-29 and Barthel Index of Daily Living Activities, 30 patients were asked to complete the questionnaires again within 10 days, and the intraclass correlation coefficients for these questionnaires were 0.78 and 0.81 , respectively. The questionnaires were completed by both groups before the intervention. Then, after the intervention, the study subjects were requested to fill out the MSIS-29 and Barthel Index of Daily Living Activities again. It should be noted that the participants in the present study and individuals who collected the questionnaires were blinded to the type of intervention.

\subsection{Statistical analysis and statistical software}

The data were analyzed by Stata software (version 13). The Kolmogorov-Smirnov test was used to investigate the distribution of the quantitative data among which the variables of age and quality of life scores (before and after the 
intervention) were reported with a normal distribution. The Chi-square test, t-test, and MannWhitney $U$ test were employed in order to analyze the demographic variables. For the analysis of daily activities, the Mann-Whitney $U$ test and Wilcoxon test were used for intergroup and intragroup analyses, respectively. To control the effect of the scores of potential confounding variables (i.e., the quality of life scores and Daily Living Activities scores before the intervention) on the quality of life score after the intervention, analysis of covariance was used, and the Cohen's d effect size was calculated to demonstrate the effect of the intervention. All the analyses were performed using both the intention-to-treat procedures. A p-value of 0.05 was considered statistically significant.

\section{Results}

In this study, the mean age of the study subjects were $40.27 \pm 8.31$ and $38.16 \pm 9.38$ years in the intervention and control groups, respectively. The mean values of disease duration were $85.42 \pm 66.45$ and $71.10 \pm 52.31$ months in the intervention and control groups, respectively. Moreover, the aforementioned differences were not statistically significant. The comparison of the two groups in terms of other variables except for the place of residence did not show a statistically significant difference between the two groups (Table 1).

A comparison of the two groups in terms of daily activity status demonstrated no significant difference between the two study groups in the pre-intervention phase. The comparison of the scores after the intervention showed the improvement of the status of daily activities in the intervention group in comparison to those of the control group (18.76 \pm 1.44 and $16.96 \pm 3.01$, respectively) with a statistically significant difference $(\mathrm{P}=0.001)$. This change was also observed in the post-intervention stage in the experimental group in comparison to that reported for the pre-intervention stage $(\mathrm{P}=0.02)$ (Table 2).

The results in Table 3 show that the mean scores of the patients' life quality after adjustment in terms of confounding variables were $66.51 \pm 15.08$ and $51.31 \pm 15.08$ in the experimental and control groups, respectively, with a statistically significant difference $(\mathrm{P}<0.001)$. Furthermore, Cohen's d of 1.01 indicated the significant effect of the intervention on the improvement of the patients' life quality.

\begin{tabular}{|c|c|c|c|c|}
\hline \multicolumn{2}{|l|}{ Variable } & Intervention group & Control group & P-value \\
\hline \multicolumn{5}{|c|}{ Continuous variable } \\
\hline \multicolumn{2}{|l|}{ Age (year) } & $40.27(8.31)$ & $38.16(9.38)$ & $0.24^{*}$ \\
\hline \multicolumn{2}{|c|}{ Disease duration (month) } & $78(84)$ & $60(66)$ & $0.33^{* *}$ \\
\hline \multicolumn{5}{|c|}{ Categorical variable n (\%) } \\
\hline \multicolumn{2}{|c|}{ Male gender } & $20(41.7)$ & $16(33.3)$ & 0.40 \\
\hline \multicolumn{2}{|l|}{ Married } & 41 (83.7) & $8(16.3)$ & 0.15 \\
\hline \multirow{3}{*}{$\begin{array}{l}\text { Educational } \\
\text { level }\end{array}$} & Illiterate & $5(10.4)$ & $6(12.0)$ & \multirow{3}{*}{0.60} \\
\hline & $\leq$ Diploma & $26(54.2)$ & $31(62.0)$ & \\
\hline & College & $17(35.4)$ & $13(26.0)$ & \\
\hline \multirow{3}{*}{$\begin{array}{l}\text { Employment } \\
\text { status }\end{array}$} & Employed & $21(46.7)$ & $20(43.5)$ & \multirow{3}{*}{0.69} \\
\hline & Unemployed & 5 (11.1) & $8(17.4)$ & \\
\hline & Housewife & $19(42.2)$ & $18(39.1)$ & \\
\hline
\end{tabular}

* Independent t-test; ${ }^{* *}$ Mann-Whitney U test; ${ }^{* *}$ Chi-square test

SD: Standard deviation; IQR: Interquartile range

Table 2. Daily Living Activities in two intervention and control groups

\begin{tabular}{lcccc}
\hline Daily Living Activities & $\begin{array}{c}\text { Intervention group } \\
\text { Median (IQR) }\end{array}$ & $\begin{array}{c}\text { Control group } \\
\text { Median (IQR) }\end{array}$ & $\mathbf{Z}$ & P-value* $^{*}$ \\
\hline Before intervention & $18(4)$ & $18.5(2)$ & -0.24 & 0.81 \\
After intervention & $19(2)$ & $18(3)$ & -3.30 & 0.001 \\
Z & -2.32 & -1.28 & & \\
P-value & 0.02 & 0.20 & & \\
\hline * Mann-Whitney U test; ${ }^{* *}$ Paired t-test; ${ }^{* *}$ Wilcoxon test & & & \\
IQR: Interquartile range & & & &
\end{tabular}

Table 3. Quality of life status between two intervention and control groups

\begin{tabular}{lccccc}
\hline Quality of life & $\begin{array}{c}\text { Before intervention } \\
\text { Mean (SD) }\end{array}$ & $\begin{array}{c}\text { After intervention } \\
\text { Adjusted mean (SD) }\end{array}$ & F & $\begin{array}{c}\text { Cohen's d } \\
\text { (95\% CI) }^{*}\end{array}$ & P-value $^{* *}$ \\
\hline Intervention group & $67.40(24.82)$ & $66.51(15.08)$ & 22.60 & 1.01 & $<0.001$ \\
Control group & $62.86(21.29)$ & $51.31(15.08)$ & & $(0.59-1.42)$ & $<0.42)$ \\
\hline
\end{tabular}

*Analysis of data using analysis of covariance, adjusted quality of life scores, Daily Living Activities scores, and location

** Cohen's d effect sizes of 0.20-0.40, 0.50-0.70, and 0.80 considered small, moderate, and large, respectively 


\section{Discussion}

The current study was carried out for the evaluation of the effect of the royal jelly capsule on the life quality of the patients suffering from MS. Loss of physical strength and reduction of daily activities leading to a decrease in the quality of life are the most important problems for the individuals suffering from MS. The obtained findings of the current study showed that there were no significant differences in the demographic characteristics of the patients in this study. One of the important points in the present study was the larger population of women which is consistent with information in many medical sources and studies. A study carried out by Maria $\mathrm{C}$ et al. on the life quality of subjects with MS showed that $72.8 \%$ of the patients were women which is in line with the results of the current study (16).

Data analysis in the present study showed that although the consumption of royal jelly had an effect on the patients' quality of life in the intervention group, the difference between the two intervention and control groups in the quality of life scores was not significant. A study carried out by Seyedi et al. indicated that the consumption of royal jelly was effective in the quality of life of postmenopausal women $(\mathrm{P}<0.001)$ (17). The findings of the aforementioned study are inconsistent with the results of the present study. One of the important reasons for the difference in the results is the difference in the study population and level of daily activities between the two studies.

The findings of the current study also showed that the level of patients' activity increased after a 90-day period of receiving the royal jelly capsule, which was significantly different in comparison to that reported for the control group. A study carried out by Mobedi et al. in 2013 examined the impact of using royal jelly on the activities of the subjects with rheumatoid arthritis and joint stiffness. The findings of the aforementioned study demonstrated that the morning joint stiffness of patients in the intervention group was reported with a greater reduction and improvement of activities than that of the patients in the control group (18). In this regard, the findings of the aforementioned study are consistent with the results of the present study. The reason for this similarity may be due to the similarity of nature (chronicity) and effect of the disease on the daily activities of patients. Similar to the results of the present study, most of the participants in the aforementioned study were women.

A study was conducted by Meng et al. on the effect of royal jelly on the muscular strength of the elderly in 2017 (13). The findings of the aforementioned study are inconsistent with the results of the current study; accordingly, the consumption of royal jelly in a 3-month period had no significant effect on the physical strength of the elderly and their daily activities. The reason for the difference in these results may be the medication dosage, participants' age, or variations in other demographic variables, such as occupation and other underlying diseases, in the two studies; in this regard, most of the participants in the present study were within the age range of 36-50 years.

Various studies show that royal jelly can be useful in autoimmune diseases; for example, Erem $\mathrm{C}$ et al. in their study indicated that the use of royal jelly can have a positive effect on patients with Graves' disease (19). In another study, Constantin et al. showed that royal jelly is useful in the treatment of lupus erythematosus due to its rich resources (20). Therefore, it can be concluded that the use of royal Jelly strengthens the immune system and will be effective in the improvement of autoimmune diseases.

According to the results of various studies on the positive effect of royal jelly on patients with neurological and autoimmune disorders and since MS is known as an autoimmune disease, it can be said that the present study has been one of the first attempts conducted on patients with MS. The current study had several limitations. Firstly, there was no information regarding the most effective dose of royal jelly for a human. It is likely necessary to use larger or smaller daily doses of royal jelly for the production of several important effects. Secondly, limited access to patients living in rural and far areas was one of the major limitations of the present study. One of the important strengths of the present study was the appropriate sample size of the study. In addition, the present study was the result of collaboration with various specialties.

\section{Conclusion}

The findings of the current study indicated the efficacy of adding royal jelly supplements to the dietary and treatment regimens of the patients. Accordingly, among the most important therapeutic goals in patients suffering from MS are the prevention of muscle weakness and reduction of daily activities and improvement of their life quality.

\section{Acknowledgements}

This study was the result of a research project with the ethics code of IR.UMSHA.REC.1397.096 obtained from the Ethics Committee of Hamadan University of Medical Sciences, Hamadan, Iran. Furthermore, the present study was registered in the Iranian Intervention Studies System (IRCT20160 110025929N13). The authors would like to express their gratitude to the Research Council and ViceChancellor of Research and Technology, Hamadan 
University of Medical Sciences (no. 9703291646), Support Association of Patients with MS, and all dear patients who assisted with this project.

\section{Footnotes}

Authors' Contribution: Kh. Oshvandi, S.Z. Masoumi, and M. Mazdeh designed the study. M. Molavi Vardanjani, M. Aghamohammadi, and F.Kazemi collected the data. Kh. Oshvandi and M. Molavi Vardanjani were involved in writing and analyzing the results. All the authors read and approved the final manuscript.

Conflict of Interests: The authors declare that there is no conflict of interest.

Ethical Approval: The present research project was approved by the Research Ethics Committee of Hamadan University of Medical Sciences (IR.UMSHA. REC.1397.96).

Funding/Support: The present study was conducted with the support of the Vice-Chancellor of Research and Technology of Hamadan University of Medical Sciences.

\section{References}

1. Bikmoradi A, Zafari A, Oshvandi K, Mazdeh M, Roshanaei G. Effect of Progressive Muscle Relaxation on Severity of Pain in Patients with Multiple Sclerosis: a Randomized Controlled Trial. Hayat. 2014 Mar 1;20(1).

2. International Multiple Sclerosis Genetics Consortium, Hafler DA, Compston A, Sawcer S, Lander ES, Daly MJ, et al. Risk alleles for multiple sclerosis identified by a genomewide study. N Engl J Med. 2007;357(9):851-62. doi: 10.1056/NEJMoa073493. [PubMed: 17660530].

3. Poorolajal J, Mazdeh M, Saatchi M, Ghane ET, Biderafsh A, Lotfi $\mathrm{B}$, et al. Multiple sclerosis associated risk factors: a casecontrol study. Iran J Public Health. 2015;44(11):1498-505. [PubMed: 26744707].

4. Etemadifar M, Sajjadi S, Nasr Z, Firoozeei TS, Abtahi SH, Akbari $\mathrm{M}$, et al. Epidemiology of multiple sclerosis in Iran: a systematic review. Eur Neurol. 2013;70(5-6):356-63. doi: 10.1159/000355140. [PubMed: 24192707].

5. Petajan JH, Gappmaier E, White AT, Spencer MK, Mino L, Hicks RW. Impact of aerobic training onfitness and quality of life in multiple sclerosis. Ann Neurol. 1996;39(4):432-41. doi: 10.1002/ana.410390405. [PubMed: 8619521].

6. Janssens AC, Doorn PV, Boer JB, van der Meché FG, Passchier J, Hintzen RQ. Impact of recently diagnosed multiple sclerosis on quality of life, anxiety, depression and distress of patients and partners. Acta Neurol Scand. 2003;108(6):389-95. doi: 10.1034/j.1600-0404.2003.00166.x. [PubMed: 14616290].
7. Freeman JA, Thompson AJ, Fitzpatrick R, Hutchinson $M$, Miltenburger C, Beckmann K, et al. Interferon-beta1b in the treatment of secondary progressive MS: impact on quality of life. Neurology. 2001;57(10):1870-5. doi: 10.1212/wnl. 57.10.1870. [PubMed: 11723278]

8. Al-Waili N, Salom K, Al-Ghamdi AA. Honey for wound healing, ulcers, and burns; data supporting its use in clinical practice. Sci World J. 2011;11:766-87. doi: 10.1100/tsw.2011.78. [PubMed: 21479349].

9. Payamani F, Nazari A, Noktehdan H, Ghadiriyan F, Karami K. Complementary therapy in patients with multiple sclerosis. Iran J Nurs. 2012;25(77):12-20.

10. Nagai T, Sakai M, Inoue R, Inoue $H$, Suzuki N. Antioxidative activities of some commercially honeys, royal jelly, and propolis. Food Chem. 2001;75(2):237-40. doi: 10.1016/S03088146(01)00193-5.

11. Barkhordari F, Taavoni S, Haghani H, Gooshe Gir A. Effect of oral Royal jelly on edema of premenstrual syndrome. Complementary Med J. 2013;3(1):355-65.

12. Morita H, Ikeda T, Kajita K, Fujioka K, Mori I, Okada H, et al. Effect of royal jelly ingestion for six months on healthy volunteers. Nutr J. 2012;11(1):77. doi: 10.1186/1475-289111-77. [PubMed: 22995464].

13. Meng G, Wang H, Pei Y, Li Y, Wu H, Song Y, et al. Effects of protease-treated royal jelly on muscle strength in elderly nursing home residents: a randomized, double-blind, placebocontrolled, dose-response study. Sci Rep. 2017;7(1):11416. doi: 10.1038/s41598-017-11415-6. [PubMed: 28900247].

14. Hobart J, Lamping D, Fitzpatrick R, Riazi A, Thompson A. The multiple sclerosis impact scale (MSIS-29) a new patient-based outcome measure. Brain. 2001;124(5):962-73. doi: 10.1093/ brain/124.5.962. [PubMed: 11335698].

15. Collin C, Wade DT, Davies S, Horne V. The Barthel ADL index: a reliability study. Int Disabil Stud. 1988;10(2):61-3. doi: 10.3109/09638288809164103. [PubMed: 3403500].

16. Ysrraelit MC, Fiol MP, Gaitán MI, Correale J. Quality of life assessment in multiple sclerosis: different perception between patients and neurologists. Front Neurol. 2018;8:729. doi: 10.3389/fneur.2017.00729. [PubMed: 29375468].

17. Seyyedi F, Kopaei MR, Miraj S. Comparison between vaginal royal jelly and vaginal estrogen effects on quality of life and vaginal atrophy in postmenopausal women: a clinical trial study. Electron Physician. 2016;8(11):3184-92. doi: 10.19082/3184. [PubMed: 28070251].

18. Mobedi Z, Soleimani F, Rafieian-Kopaei M, Parvin N, Taheri E. The effect of oral royal jelly on clinical disease activity index (CDAI) and morning stiffness in patients with rheumatoid arthritis (RA); A randomized double-blind, placebo-controlled trial. J Isfahan Med Sch. 2013;31(252):1428-34.

19. Erem C, Deger O, Ovali E, Barlak Y. The effects of royal jelly on autoimmunity in Graves' disease. Endocrine. 2006;30(2):17583. doi: 10.1385/ENDO:30:2:175. [PubMed: 17322576].

20. Constantin MM, Nita IE, Olteanu R, Constantin T, Bucur S, Matei $\mathrm{C}$, et al. Significance and impact of dietary factors on systemic lupus erythematosus pathogenesis. Exp Ther Med. 2019; 17(2):1085-90. doi: 10.3892/etm.2018.6986. [PubMed: 30679978]. 